Physics

Physics Research Publications

\title{
Bulk black hole, escaping photons, and bounds on violations of Lorentz invariance \\ S. Khlebnikov
}




\title{
Bulk black hole, escaping photons, and bounds on violations of Lorentz invariance
}

\author{
S. Khlebnikov \\ Department of Physics, Purdue University, West Lafayette, Indiana 47907, USA
}

(Received 12 January 2007; published 22 March 2007)

\begin{abstract}
There are reasons (which we enumerate) to think that an infinite extra dimension will harbor a black hole. In this case, brane-localized modes of gravity and gauge fields become quasilocalized, and light from a distant object can become extinct as it is lost to the black hole. In a concrete scenario, where the photon is localized by gravity, we find that the extinction rate for propagating photons is at least comparable to the correction to the real part of the frequency. That results, for example, in a stringent bound on renormalization of the speed of light.
\end{abstract}

DOI: 10.1103/PhysRevD.75.065021

PACS numbers: 11.10.Kk, 04.50.th, 11.30.Cp

\section{INTRODUCTION AND RESULTS}

In models with infinite extra dimensions (for a review, see Ref. [1]), the four-dimensional nature of the observable world is due to the presence of special "localized" modes for all the known particles and gravity. These modes are localized on a submanifold (now referred to as "brane") in a higher-dimensional spacetime ("bulk") [2]. One can consider localization by a scalar field [3] (a mechanism useful for producing chiral fermions) or by gravity alone [4]. A localized mode for the graviton is known to exist in the case when the bulk is locally anti-de Sitter (AdS) with a vanishing horizon [5].

Backgrounds with nonvanishing horizons are also of interest. For reasons that will be enumerated shortly, in the present paper, we consider geometries of the AdSSchwarzschild type:

$$
\begin{aligned}
d s^{2}= & -\frac{r^{2}}{R^{2}}\left(1-\frac{r_{0}^{d+1}}{r^{d+1}}\right) d t^{2}+\frac{d r^{2}}{\kappa^{2} r^{2}\left(1-\frac{r_{0}^{d+1}}{r^{d+1}}\right)} \\
& +r^{2} \sum_{i=1}^{d}\left(d \xi^{i}\right)^{2} \\
\equiv & g_{M N} d x^{M} d x^{N} .
\end{aligned}
$$

There is a black-hole horizon at $r=r_{0}$ and a brane at $r=$ $R$ (so, the extra dimension is strictly speaking not infinite, but rather "very large"); $\kappa$ is the inverse AdS radius, and the coordinates $\xi^{i}$ span a $d$-dimensional torus. Only the region $r_{0}<r \leq R$ will be important in what follows. In this region, the metric (1) solves the $(d+2)$-dimensional vacuum Einstein equations with a negative cosmological constant:

$$
\mathcal{R}_{M N}=-(d+1) \kappa^{2} g_{M N} .
$$

It is a particular case of the class of solutions described in Ref. [6].

The coordinates $\xi^{i}$ are all periodic but with different periods. Three of them $(i=1,2,3)$ are periodic with period $2 \pi$ and correspond to the three known spatial dimensions (thus $R$ has the meaning of the "size of the universe"; we take $R \sim 1 \mathrm{Gpc}$ ). The periods of the remaining $d-3$ of $\xi^{i}$ are much smaller, so these are so far unobserved compact dimensions. Note that all $d$ dimensions are warped, with the same warp factor equal to $r^{2}$.

We consider the case when the parameter $R$ is timeindependent, i.e., we do not consider cosmology of the background (1). It has been noted [7] that for $d>3$ the simplest version of such a cosmology is problematic: if all dimensions (including the compact ones) expanded at the same rate, the fine structure constant would be changing too fast. However, such a uniform expansion may be too strong a condition to assume. Indeed, the sizes of the compact dimensions are at this point arbitrary parameters (moduli of the solution). In a more complete theory, they may be set to definite values by a weak potential. We therefore consider the question of whether Eq. (1) with $d>3$ can be the late-time limit of a sensible cosmology as still open.

For $r_{0}=0$, the $d=3$ and $d=4$ versions of the metric (1) are familiar from the studies of codimension-1 (walllike) [5] and codimension-2 (stringlike) [8] branes. (In this case, the metric is often written in terms of the radial coordinate $z$ related to $r$ by $r=R e^{-\kappa z}$.) Our reasons for considering the case with the black hole $\left(r_{0}>0\right)$ are as follows.

First, there is an argument [9] that a bulk black hole must have formed at some time during cosmological history. We see no reason why that black hole should have completely evaporated by now. Second, when the number of spatial dimensions is large enough, $d>3$, the $r_{0}=0$ metric localizes gauge fields $[10,11]$. In this case, however, there is a conical singularity at $r=0$. Ways of resolving the singularity, while preserving the 4-dimensional Lorentz invariance, have been proposed [12]. On the other hand, perhaps the simplest way to get rid of the singularity is to hide it behind a black-hole horizon, as achieved by the metric (1) with $r_{0}>0$. Lorentz invariance is now broken, but the strength of this breaking is controlled by the parameter $r_{0} / R$ and is small if that parameter is small. Finally, in the extra-dimensional solution to the strong $C P$ problem [1315], instanton transitions are viewed as transport of topo- 
logical charge across the brane, with the result being "recorded" by the extra-dimensional physics. Topological charge falling into a bulk black hole (a process presumably leading to an increase in the horizon size) seems an acceptable recording mechanism.

Now, one may consider cutting the horizon away - for example, by placing a second brane at some $r=r_{-}>r_{0}$. Indeed, for $d=3$ (one extra dimension), this has been a popular framework for Lorentz-invariance breaking phenomenology [16-18]. Here, on the other hand, we consider the entire region $r_{0}<r \leq R$ and ask how the presence of the horizon affects propagation of excitations on the brane. In particular, we are interested in propagation of the transverse electromagnetic field $A_{j}$, where $j$ corresponds to one of the $\xi$ coordinates. For concreteness, we fix the boundary condition to be

$$
\left.F_{r j}\right|_{r=R}=\left.\left(\partial_{r} A_{j}-\partial_{j} A_{r}\right)\right|_{r=R}=0 .
$$

Our main goal was to compute the decay rate of the localized (now, quasilocalized) photon mode due to the photon leaking into the black hole. That such a decay rate must appear is clear from the asymptotic behavior of the mode functions near the horizon: at large values of the variable

$$
x=-\ln \left(1-\frac{r_{0}^{d+1}}{r^{d+1}}\right)
$$

the mode functions corresponding to nonzero frequencies $\omega$ go as

$$
f(x, t) \sim \exp \left\{-i \omega t \pm i \frac{\omega R x}{(d+1) \kappa r_{0}}\right\}
$$

For $\omega>0$, both modes are regular, so only continuum stable states exist. Similar considerations apply to a massless scalar (a mimic of the graviton).

In the latter case (scalar), the absence of a normalizable mode for $r_{0}>0$ (and $d=3$ ) was noted in Ref. [16]. It was interpreted there as a consequence of the geometry becoming unreliable near $r=r_{0}$ for small $r_{0}$. However, Eq. (4) applies for any $r_{0}>0$, even those for which the surface gravity at the horizon is far from the Planck scale. For such $r_{0}$, we interpret Eq. (4) as a signal that the formerly discrete localized mode now becomes a resonance with a finite decay width into the continuum. Note that for massive particles a similar effect occurs even in the absence of a black hole, i.e., for $r_{0}=0$ [19].

We find that, for all but the smallest values of the momentum, the photon dispersion law $\omega(k)$ has a sizable real part $\operatorname{Re} \omega(k) \approx \pm k$. We call this the propagating regime. More precisely, for the branch with $\operatorname{Re} \omega(k)>0$, we obtain the following results for the photon "mass" $m^{2}=$ $\omega^{2}-k^{2}$ ( $k$ is the 3 -dimensional momentum):

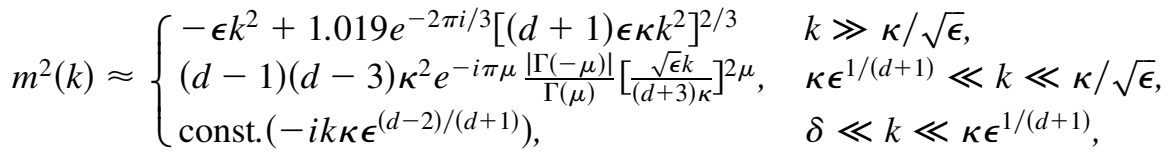

where $\mu=\frac{d-1}{d+3}, \Gamma$ is Euler's gamma function,

$$
\epsilon \equiv\left(\frac{r_{0}}{R}\right)^{d+1}
$$

is the small parameter that measures the departure from Lorentz invariance, and $\delta$ is the boundary of the propagating regime (see Sec. V for details). (The branch with $\operatorname{Re} \omega<0$, has the opposite $\operatorname{Re} \omega$ but the same $\operatorname{Im} \omega$.)

The first two lines in (5) are results of expansions in small parameters, while the third line, in which "const." is a positive numerical constant, is an order-of-magnitude estimate. The $-\epsilon k^{2}$ term in the first line (the highmomentum regime) is a trivial renormalization of the speed of light into $v=(1-\epsilon)^{1 / 2}$, due to the choice of units of length and time in Eq. (1). [In the other two regimes, this term is subleading relative to the terms included in Eq. (5).] The high-momentum regime is somewhat special: in it, the photon dissolves into a series of resonances whose widths are of the same order as the distances between them; Eq. (5) describes only the one with the smallest $|\operatorname{Im} \omega|$.
The main conclusion, then, is that, in the propagating regime, the photon decay rate

$$
\gamma(k) \equiv-2 \operatorname{Im} \omega(k) \approx-\frac{\operatorname{Im} m^{2}}{k}
$$

is at least comparable to the correction to $\operatorname{Re} \omega$, i.e., to $|\operatorname{Re} \omega(k)-v k|$.

Lorentz-noninvariant effects associated with $\operatorname{Re} \omega$, such as dependence of the speed of light on momentum or difference between the limiting speeds of different particles, will be referred to as kinematical. In the present case, the mere existence of photons that reach us from distant sources imposes stringent bounds on such effects. If photons with momentum $k$ reach us from a distance $l$, then there is a bound on $\gamma: \gamma(k) \lesssim l^{-1}$ and, in view of Eq. (5), a related bound on $|d \operatorname{Re} \omega / d k-v|$. Taking $l=1 \mathrm{Gpc}$, we obtain

$$
\left|\frac{d \operatorname{Re} \omega}{d k}(k)-v\right| \lesssim 10^{-32} \frac{\mathrm{eV}}{k} .
$$

Note that this bound is independent of any constraints on the AdS parameter $\kappa$, such as those following from the 
experimental limits [20] on power-law corrections to Newton's law. Constraints on $\kappa$, however, are useful if we want to obtain a bound on the parameter $\epsilon$ itself. For example, consider $d=5$ and use the second line in Eq. (5). In this case, $\gamma=2 \kappa \sqrt{\epsilon}$, independently of $k$. Setting $\gamma<$ $(1 \mathrm{Gpc})^{-1}$ and $\kappa>(0.1 \mathrm{~mm})^{-1}=2 \mathrm{meV}$, we obtain $\epsilon(d=5)<3 \times 10^{-60}$.

If a particle species (e.g., the electron) is tightly bound to the brane, the maximal propagation speed for it will be $v$. In this case, the bound (7) becomes a limit on the difference between that maximal speed and the speed of light. Such differences often lead to interesting effects [21], but in our case, in view of the bound (7), they look prohibitively small.

A slightly better hope for detecting a bulk black hole may be offered by the extinction effect itself. Indeed, as seen from Eq. (5), in many cases $\gamma(k)$ grows with $k$, so one can imagine a situation when the apparent loss in the luminosity of an object is negligible, say, in the optical part of spectrum but becomes significant for photons in the $\mathrm{TeV}$ range.

In the rest of the paper, after some preliminaries in Sec. II, we derive the three expressions presented in Eq. (5) (Secs. III, IV, and V). The corresponding expressions for a massless scalar are given in Sec. VI. Section VII is a brief conclusion.

\section{MODE EQUATION FOR THE PHOTON}

Equation for the electromagnetic field reads

$$
\partial_{M}\left[\sqrt{-} g g^{M N} g^{P Q} F_{N Q}\right]=0,
$$

where $g_{M N}$ is the metric extracted from Eq. (1), $F_{M N}=$ $\partial_{M} A_{N}-\partial_{N} A_{M}$, and the indices take values $0, r$, or $i$, the latter running from 1 to $d$. We begin by fixing the gauge $A_{0}=0$. Then, since the metric is static, the $P=0$ component of Eq. (8) (the Gauss law) can be written as

$$
\partial_{0} \partial_{M}\left[\sqrt{-g} g^{M N} g^{00} A_{N}\right]=0,
$$

which shows that, in addition to $A_{0}=0$, we can impose the "Coulomb gauge" condition

$$
\partial_{M}\left[\sqrt{-g} g^{M N} g^{00} A_{N}\right]=0 .
$$

Using this condition in the $P=r$ component of Eq. (8), we obtain a closed equation for $A_{r}$, which has the obvious solution $A_{r}=0$. We concentrate on this type of solutions in what follows.

The Coulomb gauge condition (9) is now simply $\partial_{i} A_{i}=$ 0 . Using this in Eq. (8) with $P=j$ and expanding $A_{j}$ in Fourier components,

$$
A_{j} \sim \exp \left(-i \omega t+i R k^{i} \xi^{i}\right),
$$

we bring the equation to the form

$$
\frac{g_{00}}{r^{d-2}} \partial_{r}\left[r^{d-2} g^{r r} \partial_{r} A_{j}\right]-k^{2}\left(R^{2} \frac{g_{00}}{r^{2}}+1\right) A_{j}=m^{2} A_{j},
$$

where $m^{2}=\omega^{2}-k^{2}$. The values of $m^{2}$ determine the photon spectrum.

In terms of the variable $x$, defined by Eq. (3), Eq. (10) becomes

$$
\begin{aligned}
- & (d+1)^{2} \frac{\kappa^{2} r_{0}^{2}}{R^{2}}\left(1-e^{-x}\right)^{(2 d-2) /(d+1)} \\
& \times \partial_{x}\left[\left(1-e^{-x}\right)^{2 /(d+1)} \partial_{x} A_{j}\right]-k^{2}\left(1-e^{-x}\right) A_{j}=m^{2} A_{j} .
\end{aligned}
$$

Equation (3) maps the range $r_{0}<r \leq R$ to the range $x_{0} \leq$ $x<\infty$. The boundary condition (2) becomes

$$
\left.\partial_{x} A_{j}\right|_{x=x_{0}}=0 .
$$

As we already mentioned, the ratio $r_{0} / R$ must be small, to ensure the smallness of deviations from Lorentz invariance on the brane. As a result,

$$
x_{0}=\epsilon+O\left(\epsilon^{2}\right),
$$

where $\epsilon$ is the small parameter (6).

The ratio appearing in front of the first term in Eq. (11),

$$
(d+1) \frac{\kappa r_{0}}{R} \equiv 2 T,
$$

has a simple physical meaning: $T(1-\epsilon)^{-1 / 2} \approx T$ is the temperature of the Hawking radiation from the black hole, as seen by an observer on the brane. [This can be deduced by continuing the metric (1) to the Euclidean time $\tau=-i t$ and requiring that the period of $\tau$ is such that the Euclidean geometry is smooth at the horizon - in the same way as the temperature was found for an AdS black hole with a spherical horizon in Ref. [22].]

We have not succeeded in solving Eq. (11) exactly. So, in what follows we consider limiting cases in which approximate expressions for $m^{2}$ can be obtained.

\section{ESCAPE NEAR THE BRANE}

We begin with the case when the photon momentum is large:

$$
\epsilon k^{2} \gg \kappa^{2},
$$

where $\epsilon$ is the parameter (6). In this case, the escape from the brane, i.e., the onset of the oscillatory behavior of the modes, occurs at $x \approx x_{0}$, and we can approximate Eq. (11) as follows:

$$
\begin{aligned}
- & 4 T^{2} x^{(2 d-2) /(d+1)} \partial_{x}\left[x^{2 /(d+1)} \partial_{x} A_{j}\right]-k^{2}\left[\epsilon+\left(x-x_{0}\right)\right] A_{j} \\
& =m^{2} A_{j} .
\end{aligned}
$$

Note that the second term on the left-hand side has been 
expanded near $x=x_{0}$, while in the first term it is sufficient to expand near zero.

A change of variables,

$$
x=\epsilon y^{d+1},
$$

converts Eq. (15) into

$$
\begin{aligned}
- & \kappa^{2} y^{d-2} \partial_{y}\left[y^{2-d} \partial_{y} A_{j}\right]-(d+1) \epsilon k^{2}\left(y-y_{0}\right) A_{j} \\
& =\left(m^{2}+\epsilon k^{2}\right) A_{j} .
\end{aligned}
$$

The range $x_{0} \leq x<\infty$ is mapped to the range $y_{0} \leq y<\infty$ with $y_{0}=1+O(\epsilon)$.

Setting $A_{j}=y^{(d-2) / 2} b$ brings Eq. (17) to the Schrödinger form:

$$
\begin{aligned}
- & \kappa^{2} \partial_{y}^{2} b+\frac{1}{4 y^{2}} d(d-2) \kappa^{2} b-(d+1) \epsilon k^{2}\left(y-y_{0}\right) b \\
& =\left(m^{2}+\epsilon k^{2}\right) b .
\end{aligned}
$$

To the accuracy indicated below, the "centrifugal" (second) term can be neglected, and the equation becomes

$$
\kappa^{2} \partial_{y}^{2} b+(d+1) \epsilon k^{2}\left(y-y_{1}\right) b=0,
$$

where

$$
y_{1}=y_{0}-\frac{m^{2}+\epsilon k^{2}}{(d+1) \epsilon k^{2}} .
$$

Solutions are the Airy functions. In accordance with the general recipe for calculating resonance energies [23], we pick the outgoing wave. For $\operatorname{Re} \omega>0$, it is

$$
A_{j}(y)=y^{(d-2) / 2} \mathrm{Ai}\left[-\alpha^{1 / 3} e^{2 \pi i / 3}\left(y-y_{1}\right)\right],
$$

where

$$
\alpha=\frac{(d+1) \epsilon k^{2}}{\kappa^{2}} .
$$

Since $\operatorname{Ai}(z)$ is an entire function, and $\alpha$ is a large parameter, the boundary condition (12) is satisfied near zeroes of $\operatorname{Ai}^{\prime}(z)$. The first zero is at $z=a_{1}^{\prime}=-1.019$. Using that, we obtain

$$
\begin{aligned}
m^{2}= & -\epsilon k^{2}+\left|a_{1}^{\prime}\right| e^{-2 \pi i / 3}\left[(d+1) \epsilon \kappa k^{2}\right]^{2 / 3} \\
& \times\left[1+O\left(\alpha^{-1 / 3}\right)+O(\epsilon)\right] .
\end{aligned}
$$

Other zeroes of $\mathrm{Ai}^{\prime}(z)$ correspond to resonances with larger $|\operatorname{Im} \omega|$.

\section{ESCAPE AT INTERMEDIATE DISTANCES}

Next, we consider cases when the inequality (14) is reversed. In low dimensionalities, $d \leq 3$, the photon is delocalized even in the absence of a black hole (for $d=$ 3 , this case was considered in Refs. [24-26]). We therefore concentrate on $d>3$, when for $r_{0}=0$ a localized mode exists $[10,11]$. For $r_{0}>0$, however, photons can escape (fall into the black hole). As we will now see, for photons with momenta in the range

$$
T \ll k \ll \frac{\kappa}{\sqrt{\epsilon}},
$$

the escape distance $y_{e}$ falls in the range

$$
1 \ll y_{e} \ll \frac{R}{r_{0}} \text {. }
$$

For $y \ll R / r_{0}, x$ is still small, and we can approximate Eq. (11) as

$$
-\kappa^{2} y^{d-2} \partial_{y}\left[y^{2-d} \partial_{y} A_{j}\right]-\epsilon k^{2} y^{d+1} A_{j}=m^{2} A_{j} .
$$

[Additional corrections - those from the higher powers of $x$ in the derivative term in Eq. (11) turn out to be negligible.]

At sufficiently small $y$, the $k^{2}$ term in (22) can be neglected, and an approximate solution can be obtained by expansion in $y$. To the required accuracy,

$$
A_{j} \approx 1+c y^{d-1}+\frac{m^{2} y^{2}}{2(d-3) \kappa^{2}},
$$

where $c$ is an integration constant. It is fixed by the boundary condition (12):

$$
c=-\frac{m^{2} y_{0}^{3-d}}{(d-1)(d-3) \kappa^{2}} .
$$

Note that, without the $k^{2}$ term, Eq. (22) is precisely the mode equation in the absence of a black hole, and indeed Eq. (23) can alternatively be obtained from the exact solution found for that case in Ref. [11].

On the other hand, for $y^{d+1} \gg\left|m^{2}\right| / \epsilon k^{2}$, we can drop the $m^{2}$ term in Eq. (22). Then, a change of variables,

$$
\zeta=\frac{2 \sqrt{\epsilon} k}{(d+3) \kappa} y^{(d+3) / 2}
$$

reduces Eq. (22) to the Bessel equation of order

$$
\mu=\frac{d-1}{d+3} .
$$

The outgoing wave (for $\operatorname{Re} \omega>0$ ) is

$$
A_{j}(\zeta)=C\left(\frac{\zeta}{2}\right)^{\mu} H_{\mu}^{(1)}(\zeta)
$$

where $H^{(1)}$ is the Hankel function, and $C$ is a constant.

Oscillations of $H^{(1)}$ set in at $\zeta \sim 1$ or, equivalently, at

$$
y \sim(\kappa / \sqrt{\epsilon} k)^{2 /(d+3)} \equiv y_{e} \gg 1 .
$$

At smaller $y$, we can use the small-argument expansion

$$
H_{\mu}^{(1)}(\zeta) \approx-\frac{i}{\pi}\left\{\Gamma(\mu)\left(\frac{\zeta}{2}\right)^{-\mu}+e^{-i \pi \mu} \Gamma(-\mu)\left(\frac{\zeta}{2}\right)^{\mu}\right\} .
$$

We see that the two terms in Eq. (26) correspond to the first two terms in (23). Therefore, to the leading order in the small parameters, $C=i \pi / \Gamma(\mu)$, and 


$$
m^{2}=-(d-1)(d-3) \kappa^{2} e^{-i \pi \mu} \frac{\Gamma(-\mu)}{\Gamma(\mu)}\left[\frac{\sqrt{\epsilon} k}{(d+3) \kappa}\right]^{2 \mu} .
$$

Note that, since $0<\mu<1, \Gamma(-\mu)$ is negative.

For the above solution to be consistent, the escape distance (25) must be much smaller than $R / r_{0}$, where the small- $x$ approximation breaks down. This leads to the left inequality in (20).

\section{ESCAPE NEAR THE HORIZON}

Photons with

$$
k \ll T
$$

escape (for $d>3$ ) at $x \gg 1$. We do not have an approximate solution that would allow us to traverse the region $x \sim 1$ and so, for this case, limit ourselves to an order-ofmagnitude estimate of $m^{2}$.

Using Eq. (23) for the region $x \ll 1$ and the outgoing wave from (4) for $x \gg 1$, and matching their logarithmic derivatives at $x=1$, we obtain

$$
m^{2}=\omega^{2}-k^{2} \sim-i \omega \kappa\left(\frac{r_{0}}{R}\right)^{d-2} .
$$

Two limits of this expression are of interest. For

$$
\kappa\left(\frac{r_{0}}{R}\right)^{d-2} \ll k \ll T,
$$

the photon is oscillating with a $k$-independent decay rate:

$$
\omega \pm k \sim-i \kappa\left(\frac{r_{0}}{R}\right)^{d-2} \text {. }
$$

In the opposite limit,

$$
k \ll \kappa\left(\frac{r_{0}}{R}\right)^{d-2} \equiv \delta,
$$

there is a mode with $\omega \sim-i \delta$, and another one with a curious diffusive behavior:

$$
\omega \sim-i \frac{k^{2}}{\delta}
$$

Thus, $k \sim \delta$ is the upper limit on the momentum of photons that can propagate on the brane.

\section{ESCAPE OF A MASSLESS SCALAR}

By the same transformations as those used in Sec. II, the equation

$$
\partial_{A}\left(\sqrt{-} g g^{A B} \partial_{B} \phi\right)=0
$$

can be brought to the form

$$
\frac{g_{00}}{r^{d}} \partial_{r}\left[r^{d} g^{r r} \partial_{r} \phi\right]-k^{2}\left(R^{2} \frac{g_{00}}{r^{2}}+1\right) \phi=m^{2} \phi,
$$

which differs from Eq. (10) for the photon only by the power in which $r$ appears in the derivative term ( $d$ instead of $d-2$ ). In terms of the variable $x$,

$$
\begin{aligned}
& -(d+1)^{2} \frac{\kappa^{2} r_{0}^{2}}{R^{2}}\left(1-e^{-x}\right)^{2 d /(d+1)} \partial_{x}^{2} \phi-k^{2}\left(1-e^{-x}\right) \phi \\
& =m^{2} \phi .
\end{aligned}
$$

We use the same boundary condition as for the vector:

$$
\left.\partial_{x} \phi\right|_{x=x_{0}}=0 .
$$

The same three cases as those in Secs. III, IV, and V can be considered. In the high-momentum case, $\epsilon k^{2} \gg \kappa^{2}$, the transformation $\phi=y^{d / 2} \chi$ results in a Schrödinger equation that differs from Eq. (18) of Sec. III only by the coefficient of the "centrifugal" term. Since that term has been dropped there anyway, the result (19) is unchanged.

For intermediate momenta, $T \ll k \ll \kappa / \sqrt{\epsilon}$, the method of Sec. IV applies. The counterpart of Eq. (22) is

$$
-\kappa^{2} y^{d} \partial_{y}\left[y^{-d} \partial_{y} \phi\right]-\epsilon k^{2} y^{d+1} \phi=m^{2} \phi .
$$

The scalar is quasilocalized for any $d>1$, a condition we now assume is satisfied. The asymptotic form (23) is replaced with

$$
\phi \approx 1+c^{\prime} y^{d+1}+\frac{m^{2} y^{2}}{2(d-1) \kappa^{2}},
$$

where $c^{\prime}$ is fixed by the boundary condition. At $y^{d+1} \gg$ $\left|m^{2}\right| / \epsilon k^{2}$, the equation is again approximately Bessel but now of order

$$
\mu^{\prime}=\frac{d+1}{d+3} .
$$

Proceeding as in Sec. IV, we obtain

$$
m^{2} \approx-\left(d^{2}-1\right) \kappa^{2} e^{-i \pi \mu^{\prime}} \frac{\Gamma\left(-\mu^{\prime}\right)}{\Gamma\left(\mu^{\prime}\right)}\left[\frac{\sqrt{\epsilon} k}{(d+3) \kappa}\right]^{2 \mu^{\prime}} .
$$

Finally, for $k \ll T$, Eq. (29) is replaced with

$$
m^{2} \sim-i \omega \kappa\left(\frac{r_{0}}{R}\right)^{d} .
$$

The higher powers of $\epsilon$ in Eqs. (33) and (34), compared to those in Eqs. (27) and (29), show that the scalar is bound to the brane tighter than the photon.

\section{CONCLUSION}

Our main (and admittedly somewhat pessimistic) conclusion is that, if our world looks like the construction described in this paper, Lorentz invariance in it is very well protected, at least at present. Indeed, in this case the rate at which a propagating photon "decays" (i.e., leaks into the black hole) is at least of the same order as the correction to the real part of the frequency. Then, the very fact that photons can propagate over astronomical distances imposes stringent bounds on kinematical violations of Lorentz invariance, such as dependence of the speed of 
light on momentum or (under mild further assumptions) difference between the limiting speeds of different particles.

In these circumstances, one may be compelled to look directly for traces of the extinction effect in astrophysical data. That, however, would seem to require a rather detailed understanding of the intrinsic luminosity of individual sources over a broad range of photon frequencies.

Finally, if the black hole has electric charge or angular momentum, these will lead to additional Lorentz- noninvariant effects, which may deserve a further investigation.

\section{ACKNOWLEDGMENTS}

The author thanks John Finley and Peter Tinyakov for discussions of the results. This work was supported in part by the U.S. Department of Energy through Grant No. DEFG02-91ER40681 (Task B).
[1] V. A. Rubakov, Usp. Fiz. Nauk 171, 913 (2001) [Phys. Usp. 44, 871 (2001)].

[2] D. W. Joseph, Phys. Rev. 126, 319 (1962).

[3] V. A. Rubakov and M. E. Shaposhnikov, Phys. Lett. B 125, 136 (1983).

[4] M. Visser, Phys. Lett. B 159, 22 (1985).

[5] L. Randall and R. Sundrum, Phys. Rev. Lett. 83, 4690 (1999).

[6] D. Birmingham, Class. Quant. Grav. 16, 1197 (1999).

[7] N. Chatillon, C. Macesanu, and M. Trodden, Phys. Rev. D 74, 124004 (2006).

[8] T. Gherghetta and M.E. Shaposhnikov, Phys. Rev. Lett. 85, 240 (2000).

[9] A. Hebecker and J. March-Russell, Nucl. Phys. B608, 375 (2001).

[10] I. Oda, Phys. Lett. B 496, 113 (2000).

[11] S. L. Dubovsky, V.A. Rubakov, and P.G. Tinyakov, J. High Energy Phys. 08 (2000) 041.

[12] E. Ponton and E. Poppitz, J. High Energy Phys. 02 (2001) 042.

[13] S. Y. Khlebnikov and M. E. Shaposhnikov, Phys. Lett. B 203, 121 (1988).

[14] S. Khlebnikov and M. Shaposhnikov, Phys. Rev. D 71, 104024 (2005).
[15] S. Khlebnikov, Phys. Rev. D 74, 085007 (2006).

[16] P. Kraus, J. High Energy Phys. 12 (1999) 011.

[17] C. Csáki, J. Erlich, and C. Grojean, Nucl. Phys. B604, 312 (2001).

[18] J.M. Cline and L. Valcárcel, J. High Energy Phys. 03 (2004) 032.

[19] S. L. Dubovsky, V. A. Rubakov, and P. G. Tinyakov, Phys. Rev. D 62, 105011 (2000).

[20] C. D. Hoyle, D. J. Kapner, B. R. Heckel, E. G. Adelberger, J.H. Gundlach, U. Schmidt, and H.E. Swanson, Phys. Rev. D 70, 042004 (2004).

[21] S. R. Coleman and S.L. Glashow, Phys. Rev. D 59, 116008 (1999).

[22] S. W. Hawking and D. N. Page, Commun. Math. Phys. 87, 577 (1983).

[23] L.D. Landau and E. M. Lifshitz, Quantum Mechanics: Non-relativistic Theory (Nauka, Moscow, 1974), 3rd ed., Sec. 134.

[24] H. Davoudiasl, J. L. Hewett, and T. G. Rizzo, Phys. Lett. B 473, 43 (2000).

[25] A. Pomarol, Phys. Lett. B 486, 153 (2000).

[26] B. Bajc and G. Gabadadze, Phys. Lett. B 474, 282 (2000). 